Editorial

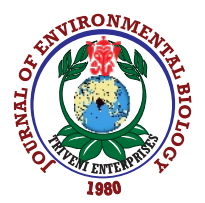

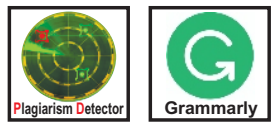

\title{
Immunology of COVID-19 and its clinical implications for therapy
}

The world has already experienced several viral attacks in the $21^{\text {st }}$ Century (Christou, 2011) have affected millions of people globally and caused substantial mortality and morbidity (Sutton, 2018). One that caused global pandemic and fear is newly emerged novel coronavirus (COVID-19) (Wilder-Smith et al., 2020). It is highly contagious, large enveloped, single-stranded, nonsegmented, positive-sense RNA viruse that belong to coronaviridae family and genus Beta coronavirus (Yan et al., 2020). The viruses of coronaviridae family are further divided into two subfamilies; Middle East respiratory syndrome coronavirus (MERS$\mathrm{CoV}$ ) and severe acute respiratory syndrome coronavirus (SARS-CoV) (Kindler et al., 2016). COVID-19 show close similarities to SARS-CoV due to phylogenetic relationship and genome structure. Word Health Organisation (WHO) has designated the current pandemic of SARS-CoV-2 infection as COVID-19 (Sohrabi et al., 2020). The virus shows high infectivity and mainly transmitted in the form of droplets with an incubation period of about 4-5 days. The onset of symptoms in patients is usually within 11-12 days (Linton et al., 2020). The most prominent symptoms seen in almost every patient includes dry cough, fever, fatigue, sore throat, lack of smell and taste, and severe patients suffer from breathing difficulties (Guan et al., 2019). We do not have any effective therapeutics or drugs approved by the U.S. Food and Drug Administration (FDA) to prevent or treat COVID-19 infections presently. The only treatment available for patients with COVID-19 is supportive care, including supplement oxygen and mechanical ventilator support. Therefore, there is an urgent need to develop an effective therapy to treat patients infected with COVID-19. The present situation calls much attention on prophylactics and therapeutics approach.

This virus contain four structural proteins viz. the spike $(S)$, membrane(M),envelop (E) and nucleocapsid $(N)$. The $S$ protein is considered to be highly immunogenic and contribute protective immunity in contrast to N, M and E proteins(Saha et al., 2020). Many laboratories have confirmed the entry of virus into human cells after its fusion with cell membrane of target tissues. After fusion, the $S$ protein undergoes proteolysis by host proteases and give rise to $\mathrm{S} 1$ and $\mathrm{S} 2$ fragments. Thus, $\mathrm{S} 1$ protein is ready to interact with angiotensin converting 2 (ACE2) receptors present on respiratory and intestine epithelia besides alveolar macrophages present in the lungs(Cheng et al., 2020). Few studies have confirmed that ACE2 receptors are present in other cells too. S1 protein has a domain that interacts specifically with a domain on the ACE2 receptor. This interaction is must to produce the symptoms of disease (Nadeem et al., 2020). One must understand the role of chloroquine analogues works here by inhibiting virus fusion into the cell membrane. Some laboratories have generated both polyclonal and monoclonal antibodies against $\mathrm{S} 1$ domain and inhibited the interaction (Ravichandran et al., 2020; Zhao et al., 2020). This is one of the areas of immunotherapy. Some key amino acids present on the S1 domain initiate this interaction with high affinity (Saha et al., 2020). If these amino acids are blocked by monoclonal antibodies or lacked by chance due to mutation, the rate of infection would be reduced and patients may show minimal symptoms. This protective effect can be seen by reduced affinity of viruses to ACE2 receptor that would result in decreased infection.

As the virus is getting mutated, some infected persons shown critical symptoms and other escape the disease severity. If the virus continues to mutate to lower its pathogenicity, there is a high possibility that it might coexist with humans. Using computational and bioinformatics, few laboratories have predicted $B$ cells, $T$ cells and cytotoxic $T$ cells epitopes on $S$ protein as well as other structural proteins(Baruah et al., 2020). This will help us to develop virus specific neutralizing antibodies by gene cloning or peptide synthesisbased approach. We personally feel if we have antisera from recovered patients of COVID-19 infection, using panel of peptides from different regions of viral proteins will help us to develop diagnosis kits and peptide-based vaccine. Since immune response is based on individual Human Leukocytes Antigens (HLA), the above approach will help to identify immunodominant regions on viral proteins. In addition, we can identify the polymorphic loci on HLA genes among infected persons showing clinical symptoms and others escape the disease severity to confirm the susceptibility towards infection, immunity and outcomes.

COVID-19 infection has led to inflammatory storm due to presence of hyper activated immune cells and over-production of proinflammatory cytokines that may determine the outcome (Ye et al., 2020). Several studies have shown the presence of elevated levels of 
serum pro-inflammatory cytokines (IL-6, IL-1 $\beta$ and TNF- $\alpha$ ) and increased immune inflammatory cells leading to cytokine storm, which could be partially responsible for immune mediated problems in the lungs of the patients(Ye et al., 2020; Felsenstein et al., 2020). Immune mediated, acute respiratory distress syndrome (ARDS) are commonly seen in ICU that are associated with poor outcome in patients with COVID-19 (Zhang et al., 2020). Therefore, specific blockade of inflammatory storm associated with hyper-inflammation during COVID-19 is an important therapeutic approach when compared to the systemic immunosuppression. To our knowledge, few clinical trials are ongoing to test the safety and efficacy of targeted therapies associated with IL-6, IL-1 receptor and Janus kinases (JAK) blockade in severe COVID-19 patients (Neurath, 2020; Russell et al., 2020). Like targeted therapies, several clinical trials of anti-viral drugs therapies such as Remdesivir, Lopinavin and Favipiravin are ongoing study (Şimşek and Ünal, 2020; Cao et al., 2020). The safety and efficacy of these trials are still unclear which need furthermore investigations in patients across the globe. Recently, few studies have confirmed blood clots in lungs and heart of patients with COVI-19 (Connors and Levy, 2020). We feel that treatment with blood thinner or low molecular weight heparin can reduce the problems of blood clots.

Development of animal models of coronavirus infection are useful for testing vaccines and antiviral drugs. However, currently available animal models do not reproduce the inflammatory storm or pulmonary disease observed in individuals infected with SARS-CoV (Cleary et al., 2020). SARS-CoV viruses has been found to replicate in the lungs of domestic cats, hamsters, and mice. These animal models remain symptomatic (Tiwari et al., 2020). Therefore, we believe that development of animal models that establishes COVID-19 infections can show symptoms require a transgenic animal approach. The knowledge obtained from the study of animal models will help us in developing specific therapies, and vaccine would minimize pulmonary disease and optimise the inflammatory storm. Finally, we conclude that vaccine is the best for protection against COVID-19 infections, but development of antiviral drugs or targeted therapies are urgently required to cure COVID-19 patients.

\section{References}

Baruah, V. and S. Bose: Immunoinformatics-aided identification of T cell and B cell epitopes in the surface glycoprotein of 2019-nCoV. J. Med. Virol,, 92, 495-500 (2020).

Cao, B., Y. Wang, D. Wen, W. Liu, J. Wang, G. Fan, L. Ruan, B. SongB, Y. Cai, M. Wei, X. Li, J. Xia, N. Chen, J. Xiang, T. Yu, T. Bai, X. Xie, L. Zhang, C. Li, Y. Yuan, H. Chen, H. Li, H. Huang, S. Tu, F. Gong, Y. Liu, Y. Wei, C. Dong, F. Zhou, X. Gu, J. Xu, Z. Liu, Y. Zhang, H. Li, L. Shang, K. Wang, K. Li, X. Zhou, X. Dong, Z. Qu, S. Lu, X. Hu, S. Ruan, S. Luo, J. Wu, L. Peng, F. Cheng, L. Pan, J. Zou, C. Jia, J. Wang, X. Liu, S. Wang, X. Wu, Q. Ge, J. He, H. Zhan, F. Qiu, L. Guo, C. Huang, T. Jaki, F.G. Hayden, P.W. Horby, D. Zhang and C. Wang: A trial of Lopinavir-Ritonavir in adults hospitalized with severe Covid-19. N. Engl. J. Med., 382, 1787-1799 (2020).

Cheng, H., Y. Wang and G.Q. Wang: Organ-protective effect of angiotensin-converting enzyme 2 and its effect on the prognosis of COVID-19. J. Med. Virol., 92, 726-730 (2020).

Christou, L.: The global burden of bacterial and viral zoonotic infections. Clin. Microbiol. Infect., 17,326-330 (2011).

Cleary, S.J., S.C. Pitchford, R.T. Amison, R. Carrington, C.L.R. Cabrera, M. Magnen, M.R. Looney, E. Gray and C. P. Page: Animal models of mechanisms of SARS-CoV-2 infection and COVID-19 pathology. Br. J. Pharmacol., 177, 4851-4865 (2020).

Connors, J.M. and J.H. Levy: COVID-19 and its implications for thrombosis and anticoagulation. Blood, 135, 2033-2040 (2020).

Felsenstein, S., J.A. Herbert, P.S. McNamara and C.M. Hedrich: COVID-19: Immunology and treatment options. Clin. Immunol., 215, 108448 (2020).

Guan, W., Z. Ni, Y. Hu, W. Liang, C. Ou, J. He, L. Liu, H. Shan, C. Lei, D.S.C. Hui, B. Du, L. Li, G. Zeng, K.-Y. Yuen, R. Chen, C. Tang, T. Wang, P. Chen, J. Xiang, S. Li, Jin-lin Wang, Z. Liang, Y. Peng, L. Wei, Y. Liu, Ya-hua Hu, P. Peng, Jian-ming Wang, J. Liu, Z. Chen, G. Li, Z. Zheng, S. Qiu, J. Luo, C. Ye, S. Zhu, and N. Zhong: Clinical characteristics of Coronavirus disease 2019 in China. N. Engl. J. Med., 382, 1708-1720 (2020).

Kindler, E, V. Thiel and F. Weber: Interaction of SARS and MERS Coronaviruses with the antiviral interferon response. Adv. Virus Res., 96, 219-243 (2016).

Linton, N.M., T. Kobayashi, Y. Yang, K. Hayashi, A.R. Akhmetzhanov, S. Jung, B. Yuan, R. Kinoshita and H. Nishiura: Incubation period and other epidemiological characteristics of 2019 Novel Coronavirus infections with right truncation: A statistical analysis of publicly available case data. J. Clin. Med., 9, 538 (2020). doi: 10.3390/jcm9020538

Nadeem, M.S., M.A. Zamzami, H. Choudhry, B.N. Murtaza, I. Kazmi, H. Ahmad and A. R. Shakoori : Origin, potential therapeutic targets and treatment for Coronavirus disease (COVID-19). Pathogens., 9, (2020). doi: 10.3390/pathogens9040307

Neurath, M.F.: COVID-19 and immunomodulation in IBD. Gut. 69, 1335-1342 (2020).

Ravichandran, S., E.M. Coyle, L. Klenow, J. Tang, G. Grubbs, S. Liu, T. Wang, H. Golding and S. Khurana: Antibody signature induced by SARS-CoV-2 spike protein immunogens in rabbits. Sci. Transl. Med., 12, eabc3539 (2020).

Russell, B., C. Moss, G. George, A. Santaolalla, A. Cope, S. Papa and M. V. Hemelrijck: Associations between immune-suppressive and stimulating drugs and novel COVID-19-a systematic review of current evidence. Ecancermedicalscience., 14, 1022 (2020).

Saha, P., A.K. Banerjee, P.P. Tripathi, A.K. Srivastava and U. Ray: A virus that has gone viral: amino acid mutation in S protein of Indian isolate of Coronavirus COVID-19 might impact receptor binding, and thus, infectivity. Biosci. Rep., 40, BSR20201312 (2020). 
Şimşek, Y. S. and S. Ünal. Antiviral treatment of COVID-19. Turk. J. Med. Sci., 50,611-619 (2020).

Sohrabi, C., Z. Alsafi, N. O'Neill, M. Khan, A. Kerwan, A. Al-Jabir, C. losifidis and R. Agha: World Health Organization declares global emergency: Areview of the 2019 novel coronavirus (COVID-19). Int. J. Surg., 76, 71-76 (2020).

Sutton, T.C.: The pandemic threat of emerging H5 and H7 avian influenza viruses. Viruses, 10, 461 (2018). doi: 10.3390/v10090461

Tiwari, R., K. Dhama, K. Sharun, M. I. Yatoo, Y. S. Malik, R. Singh, I. Michalak, R. Sah, D. K. Bonilla-Aldana and A. J. Rodriguez-Morales: COVID-19: animals, veterinary and zoonotic links. Vet. Q., 40, 169-182 (2020).

Wilder-Smith, A., C.J. Chiew and V.J. Lee: Can we contain the COVID-19 outbreak with the same measures as for SARS? Lancet Infect. Dis., 20, e102-e107 (2020).

Yan, R., Y. Zhang, Y. Li, L. Xia, Y. Guo and Q. Zhou: Structural basis for the recognition of SARS-CoV-2 by full-length human ACE2. Science, $367,1444-1448(2020)$

Ye, Q., B. Wang and J. Mao: The pathogenesis and treatment of the 'Cytokine Storm' in COVID-19. J. Infect., 80, 607-613 (2020).

Zhang, X., S. Li and S. Niu:ACE2 and COVID-19 and the resulting ARDS. Postgrad. Med. J., 96, 403-7 (2020).

Zhao, R., M. Li, H. Song, J. Chen, W. Ren, Y. Feng, G. F. Gao, J. Song, Y. Peng, B. Su, X. Guo, Y. Wang, J. Chen, J. Li, H. Sun, Z. Bai, W.J. Cao, J. Zhu, Q. Zhang, Y. Sun, S. Sun, X. Mao, J. Su, X. Chen, A. He, W. Gao, R. Jin, Y. Jiang and L. Sun: Early detection of SARS-CoV-2 antibodies in COVID-19 patients as a serologic marker of infection. Clin. Infect. Dis., ciaa523 (2020). doi: 10.1093/cid/ciaa523

Dr. D. N. Rao (Corresponding author)

Former (Professor \& Head), Department of Biochemistry

All India Institute of Medical Science (AlIMS), New Delhi, India

President of Indian Immunology Society (Ex)

Email: dnra0311@rediffmail.com

\section{Dr. Dablu Lal Gupta}

Assistant Professor

Institute of Science, Nirma University

Ahmedabad, Gujarat, India

Email: dablulal.gupta@nirmauni.ac.in 